Ann. Biol. anim. Bioch. Biophys., I974, 14 (I), I57-I65.

\title{
INFLUENCE DE LA DÉFAUNATION SUR L'UTILISATION DE LA SÉLÉNOMÉTHIONINE CHEZ LE MOUTON
}

\author{
M. HIDIROGLOU et $K$. J. JENKINS \\ avec la collaboration technique de E. S. QuirtKaT \\ Institut de Recherches zootechniques, \\ Ministère de l'A griculture. \\ Ottawa (Canada) \\ RÉSUMÉ
}

L'étude de l'utilisation de la sélénométhionine chez le Mouton défauné ou non a été réalisée après son marquage au ${ }^{25} \mathrm{Se}$ ou au ${ }^{3} \mathrm{H}$. Vingt moutons ont été divisés en 2 groupes de Io chacun; le premier a été constitué d'individus normaux, et le second d'animaux défaunés. La défaunation a été réalisée en administrant oralement, pendant 6 jours consécutifs, du dioctylsulphosuccinate de sodium, lequel a permis d'éliminer complètement les protozoaires dès les trois premiers jours du traitement. Dans chaque groupe de Io animaux, six ont reçu du ${ }^{75}$ Se et quatre du tritium. La répartition de la radioactivité tissulaire et son excrétion n'ont démontré aucune différence significative due à la défaunation. Des radioactivités maximales ont été enregistrées dans les reins, foie, pancréas ; on a noté également de hautes activités spécifiques dans certaines parties du tube digestif. Le pic de la radioactivité plasmatique, après l'administration de radiosélénium, s'est situé à 24 heures pour le ${ }^{75} \mathrm{Se}$ et 60 heures pour le tritium ; des radioactivités maximales urinaires apparaissaient 24 heures après l'ingestion des radioisotopes. Il a été remarqué que plus de radioactivité a été excrétée par les fèces que par les urines. Un essai a été également entrepris en vue d'étudier le métabolisme de la ${ }^{75}$ Se-sélénométhionine dans le rumen de cinq autres moutons. Les résultats ont démontré que seulement i 8 p. Ioo de la radioactivité des protéolysats bactériens du rumen chromatographiés étaient retrouvés incorporés dans la sélénométhionine.

\section{INTRODUCTION}

Il existe dans le rumen une population immense de protozoaires ciliés dont le nombre, d'après HUNGATE (I950) dépasserait le million par $\mathrm{g}$ de contenu de rumen. Cependant si leur morphologie détaillée est décrite chez les ruminants depuis long-

Contribution No. 483 Animal Research Institute, Ottawa, Canada. 
temps, par contre les travaux expérimentaux consacrés à leur rôle physiologique sont relativement restreints. Ces micro-organismes, qui contiennent environ $20 \mathrm{p}$. Ioo de l'azote total du rumen (WELLER et coll, I958) jouent un rôle fort utile dans la rétention de cet élément chez les ruminants (ABOU AKKadA et El-ShazLy, I965). Si ces ciliés concourent, d'après HARMEYER et HiLL (I965), à la synthèse des acides aminés dans le rumen, ils sont également capables, selon CoLEMAN (I967), WILLIAMs et coll. (I96I), d'utiliser directement certains acides aminés libres. La méthode de défaunation chimique simple des bovins découverte récemment par AвоU AkKADA et coll. (I968) nous a incité à étudier le métabolisme d'un acide aminé sélénié tel que la sélénomé thionine chez le mouton possédant ou non une telle faune.

\section{MATÉRIEL ET MÉTHODES}

\section{Animaux}

Les expériences ont été effectuées sur 20 moutons de $24-30 \mathrm{~kg}$ de poids vif chacun placés, dès le début de l'expérience, dans des cages à métabolisme.

Ces animaux ont été utilisés par paire, en ro essais successifs, et à chaque essai, 2 animaux ont été gardés en chambres séparées et soignés par deux personnes différentes. De cette façon il n'y a eu aucun contact physique entre les animaux. Pendant six jours consécutifs, on administra par voie orale, à un mouton de chaque paire, $5 \mathrm{~g}$ de dioctylsulphosuccinate de sodium par $30 \mathrm{~kg}$ de poids vifs, Cyanamid of Canada, Montréal) répartis équitablement en 2 doses, une le matin et l'autre le soir. Durant les huit jours qui ont suivi la dernière administration de ce produit en vue de la défaunation du mouton, des prélèvements du contenu du rumen ont été effectués au moyen d'une sonde cesophagienne.

L'examen microscopique de ces prélèvements en vue de déceler les protozoaires ont été effectués suivant la méthode de Sauvant et Gouet (1970). A la fin de ce huitième jour, on a administré le radioisotope dans le rumen de chaque mouton au moyen d'une sonde osophagienne. Six paires de moutons ont ainsi reçu de la ${ }^{75}$ Se-sélénométhionine $(20 \mu \mathrm{C} / \mathrm{kg}$ de poids vif $)$ tandis que les quatre autres paires recevaient du DL-sélénométhionine-méthyl- $\mathrm{T}$ ( $4 \circ \mu \mathrm{Ci} / \mathrm{kg}$ de poids vif). En outre, on a administré par voie orale à cinq autres moutons de $30-35 \mathrm{~kg}$ de poids vif une seule dose de ${ }^{75}$ Sesélénométhionine $(7,14 \mathrm{Ci} / \mathrm{kg}$ de poids vif). Tout au long des 96 heures qui ont suivi l'administration de ce radioisotope, des prélèvements successifs du contenu du rumen ont été effectués au moyen d'une sonde œsophagienne. Les bactéries du rumen ont été séparées des protozoaires et des débris alimentaires selon la technique de WRIGHT et Hungate (1967).

\section{Analyses effectuées}

Les bactéries du rumen ont subi les transformations suivantes : précipitation avec l'acide trichloroacétique (ATA) Io p. Ioo, lavage du sédiment à l'ATA, à deux reprises, puis trois fois à l'éther, lequel par la suite a été évaporé complètement sous azote. Le sédiment a été ensuite hydrolysé soit par la pronase (HEDEGAARD et col, I963; ConTI et coll., 1972) soit au moyen d'acide chlorhydrique $6 \mathrm{~N}$ sous vide à $100{ }^{\circ} \mathrm{C}$ pendant I 8 heures. Les hydrolysats contenant le ${ }^{75}$ Se ont été séparés par chromatographie sur papier. Les techniques chromatographiques et autoradiographiques, ainsi que la mesure de la radioactivité des acides aminés marqués ont été décrits antérieurement. (Jenkins et Hidiroglou, i967; Hidiroglou, Heaney et Jenkins, I968).

\section{Mesure de la radioactivité.}

Le sang, l'urine et les fèces ont été prélevés aux heures indiquées dans les tableaux. Les moutons ont été tués Io8 heures après l'administration du radioisotope.

Des échantillons de tissus lyophilisés (environ $\mathrm{I}$ o $\mathrm{mg}$ ) des moutons administrés à la DL-sélénométhionine-méthyl-T, activité spécifique I.3 curies $/$ mmole, ont été dissous avec du Protosol (New England Nuclear, Boston). Des échantillons frais de plasma et d'urine ont été également 
dissous avec du Biosolv BBS-3 (Beckman) ; la radioactivité du tritium a été mesurée suivant une technique proposée par PATTERSON et GREENF (1965), à l'aide d'un compteur à scintillation liquide Beckman LS-25o. L'activité spéci fique qui est rapportée en $\mathrm{dpm} / \mathrm{g}$ de tissu lyophilisé est obtenue par l'emploi d'étalons internes. Les radioactivités des échantillons de plasma, et de tissus frais des moutons dosés à la ${ }^{75}$ Se-sélénométhionine (activité spécifique I-ro $\mathrm{mCi} / \mathrm{mg}$ sélénométhionine), ont été mesurées avec un compteur Packard autogamma spectromètre modèle 578 .

\section{Analyses statistiques.}

Elles ont été conduites au moyen de l'analyse de la variance. Étant donné que l'écart-type de la moyenne était proportionnel à la moyenne, il s'est avéré nécessaire de se servir de la transformation logarithmique afin de stabiliser la variance (SNEDECOR et Cochran, 1967). A la suite de ces transformations, les écarts-types ont été plus normalement distribués autour de la moyenne.

\section{RÉSULTATS ET DISCUSSION}

\section{Examen microscopique $d u$ contenu $d u$ rumen}

L'emploi du dioctylsulphosuccinate de sodium (Dossna) a permis d'éliminer complètement, dès la première administration, la plus grande partie des infusoires ciliés tel que Epidinium caudatum et Ophryoscolex caudatum, tandis que 2-3 doses ont été nécessaires pour s'assurer de l'élimination des petits oligotriches tel que Entodinium sp. Ces résultats concordent avec ceux que DAwson et KEMP (I969) ont relevé chez le Mouton lors de la défaunation.

Il y a lieu de mentionner que ce produit a bien été toléré par nos animaux, lesquels n'ont présenté aucun signe d'intoxication. Ceci est probablement dû au fait que ce produit a été administré en deux demi-doses journalières. Une observation analogue a d'ailleurs été relevée par L,INDSAy et Hogan (I972).

\section{Radioactivité dans les tissus}

Les résultats d'ensemble sont consignés dans le tableau I. Chez les moutons ayant reçu l'un ou l'autre radioisotope, on a constaté que s'il y avait plus de radioactivité dans les tissus des moutons défaunés, la différence avec les contrôles n'était cependant pas significative $(\mathrm{P}>0,05)$. I1 ressort aussi qu'après l'administration de l'un ou l'autre de ces radioisotopes, des radioactivités particulièrement élevées ont été enregistrées dans les pancréas, foie et reins. Ceci confirme les résultats obtenus par NeETHLING et coll. (I968). Mentionnons également que des activités spécifiques très élevées ont été enregistrées dans les caillette et duodénum, tissus dont les muqueuses sont caractérisées par une synthèse protéique rapide. Cette observation confirme celles de Jacobson (Ig66) et Hanson, Jacobson (Ig66), sur le Rat. A l'encontre de ces auteurs, les concentrations des radioactivités sont moins élevées dans le coeur et les muscles. A la lecture de ce tableau, il est à remarquer que si la concentration de la radioactivité dans la bile des animaux traités au radioisotope tritié peut être considérée relativement haute par rapport à celle des autres tissus, elle devient faible avec le radioisotope sélénié ; l'inverse se produit pour la rate. 


\section{TABLEAU I}

Distribution de la radioactivité parmi les divers tissus des moutons (1) quatve jours apvès l'administration orale d'une seule dose de ${ }^{75}$ Se-L-sélénométhionine (2o $\mu \mathrm{Ci} / \mathrm{kg}$ de poids vif) ou $D L$-sélénométhionine (méthyl-T) (40 $\mu \mathrm{Ci} / \mathrm{kg}$ de poids vif)

Les valeurs sont données en logarithmes coups/mn/g de tissu frais pour le ${ }^{75}$ Se et logarithmes désintégration/mn/g de tissu lyophilisé pour le ${ }^{3} \mathrm{H}$

Radioactivity in tissues of wethers (1)

four days after administration of a single oral dose of ${ }^{75}$ Se-L-sélénométhionine

(20 $\mu \mathrm{Ci} / \mathrm{kh}$ body weight) or $D L$-sélénométhionine (methyl-T) (4o $\mu \mathrm{Ci} / \mathrm{kg}$ body weight)

Values are mean logarithms of $\mathrm{cpm} / \mathrm{g}$ of fresh tissue for the ${ }^{75} \mathrm{Se}$ and mean logarithms of disintegration $/ \mathrm{mn} / \mathrm{g}$ freeze dry tissue for the ${ }^{3} \mathrm{H}$

\begin{tabular}{|c|c|c|c|c|c|c|c|}
\hline \multirow{2}{*}{\multicolumn{2}{|c|}{$\begin{array}{c}\text { Tissue } \\
\text { Location of } \\
\text { Tissue Sample }\end{array}$}} & \multicolumn{3}{|c|}{${ }^{75}$ Se-sélénométhionine } & \multicolumn{3}{|c|}{${ }^{3} \mathrm{H}$-sélénométhionine } \\
\hline & & \multirow{2}{*}{$\mid \begin{array}{c}\text { Sans infusoires } \\
\text { Defaunated }\end{array}$} & \multirow{2}{*}{$\begin{array}{c}\begin{array}{c}\text { Holoxéniques } \\
\text { Control }\end{array} \\
4,50\end{array}$} & \multirow{2}{*}{$\begin{array}{l}\text { ET } \\
\text { SE } \\
0,11\end{array}$} & \multirow{2}{*}{$\begin{array}{c}\begin{array}{c}\text { Sans infusoires } \\
\text { Defaunated }\end{array} \\
2,45\end{array}$} & \multirow{2}{*}{$\begin{array}{c}\begin{array}{c}\text { Holoxéniques } \\
\text { Control }\end{array} \\
2,\}_{\mathbf{t}} 3\end{array}$} & \multirow{2}{*}{$\begin{array}{l}\text { ET* } \\
\text { SE } \\
0,01\end{array}$} \\
\hline Rumen & Rumen & & & & & & \\
\hline Réseau & Reticulum & 4,73 & 4,43 & 0,09 & 2,56 & 2,62 & 0,06 \\
\hline Feuillet & Omasum & 4,52 & 4,41 & 0,05 & 2,56 & 2,56 & 0,01 \\
\hline Caillette & Abomasum & 4,59 & 4,61 & 0,08 & 2,75 & 2,68 & 0,08 \\
\hline Duodénum & Duodenum & 4,72 & 4,62 & 0,09 & 2,73 & 2,71 & 0,01 \\
\hline Côlon & Colon & 4,61 & 4,43 & 0,08 & 2,62 & 2,65 & 0,02 \\
\hline Foie & Liver & 4,82 & 4,71 & 0,07 & 3,00 & 3,00 & 0,01 \\
\hline Pancréas & Pancreas & 5,21 & 5,01 & 0,12 & 2,87 & 3,03 & 0,33 \\
\hline Rate & Spleen & 4,82 & 4,61 & 0,09 & 2,69 & 2,53 & 0,73 \\
\hline Bile & Bile & 3,22 & 3,20 & 0,11 & 2,74 & 2,71 & 0,03 \\
\hline $\begin{array}{l}\text { Capsules } \\
\text { surrénales }\end{array}$ & Adrenals & - & - & - & 2,72 & 2,85 & 0,87 \\
\hline Reins & Kidney & 5,69 & 5,60 & 0,08 & 2,82 & 2,95 & 0,38 \\
\hline Poumons & Lung & 4,54 & 4,36 & 0,09 & 2,50 & 2,54 & 0,12 \\
\hline Muscle & Muscle & 4,00 & 3,86 & 0,12 & 2,29 & 2,39 & 0,23 \\
\hline Cour & Heart & 4,30 & 4,18 & 0,10 & 2,41 & 2,52 & 0,60 \\
\hline Graisses & Depot Fat & 3,75 & 3,61 & 0,17 & - & - & - \\
\hline
\end{tabular}

(1) 20 moutons de $2{ }^{2}-30 \mathrm{~kg}$ de poids vif ont sarvis 6 dans chaque traitements ${ }^{75}$ Se et 4 pour chaque traitements ${ }^{3} \mathrm{H}$.

20 sheep of $24-30$ body weight used -6 in each ${ }^{75}$ Se-treatment and 4 for each ${ }^{3} \mathrm{H}$-treatment.

* ET = Écart type

$\mathrm{SE}=$ Standard error

\section{Radioactivité dans le plasma}

Nous avons rassemblé les résultats des radioactivités plasmatiques dans le tableau 2. La première constatation qui s'impose est que si les radioactivités sont légèrement plus hautes pour les défaunés, la différence n'est cependant jamais significative $(P>0,05)$. Le pic de radioactivité plasmatique des moutons ayant reçu du ${ }^{75}$ Se s'est situé à $24 \mathrm{~h}$. alors que chez les animaux traités au tritium, il survenait à $60 \mathrm{~h}$. 


\section{TABLEAU 2}

Évolution des radioactivités dans le plasma des moutons ( $\left.{ }^{(}\right)$ après administration orale d'une seule dose de ${ }^{75} \mathrm{Se}$ ou $"{ }^{3} \mathrm{H}$-sélénométhionine

Les valeurs sont données respectivement en logarithmes $\left({ }^{75} \mathrm{Se}\right) \mathrm{cpm} / \mathrm{ml}$ ou $\left({ }^{3} \mathrm{H}\right) \mathrm{dpm} / \mathrm{ml}$ de plasma

Radioactivity in plasma of wethers ( $\left.{ }^{1}\right)$

after administratory of a single oral dose of ${ }^{75} \mathrm{Se}$ or $"{ }^{3} \mathrm{H}$-sélénométhionine

Values are mean logarithms of $\left({ }^{75} \mathrm{Se}\right) \mathrm{cpm} / \mathrm{ml}$ or $\left({ }^{3} \mathrm{H}\right) \mathrm{dpm} / \mathrm{ml}$ of plasma

\begin{tabular}{|c|c|c|c|c|c|c|}
\hline \multirow{2}{*}{\begin{tabular}{c} 
Temps \\
(heures) $\quad$ Time \\
après hours) \\
administration \\
\multicolumn{2}{c}{${ }^{\mathbf{7 5}} \mathrm{Se}$}
\end{tabular}} & \multicolumn{3}{|c|}{${ }^{75}$ Se-sélénométhionine } & \multicolumn{3}{|c|}{${ }^{3} \mathrm{H}$-sélénométhionine } \\
\hline & $\begin{array}{c}\text { Sans infusoires } \\
\text { Defaunated }\end{array}$ & $\begin{array}{c}\text { Holoxéniques } \\
\text { Control }\end{array}$ & $\begin{array}{l}\mathrm{ET} \\
\mathrm{SE}\end{array}$ & $\begin{array}{c}\text { Sans infusoires } \\
\text { Defaunated }\end{array}$ & $\begin{array}{c}\text { Holoxéniques } \\
\text { Control }\end{array}$ & $\begin{array}{l}\mathrm{ET} \\
\mathrm{SE}\end{array}$ \\
\hline 1 & 3,23 & 3,07 & 0,10 & 3,99 & 3,94 & 0,05 \\
\hline 2 & 3,70 & 3,14 & 0,16 & 4,08 & 4,02 & 0,05 \\
\hline 3 & 3,81 & 3,57 & 0,15 & 4,03 & 4,05 & 0,05 \\
\hline 4 & 3,98 & 3,78 & 0,15 & 4,12 & 4,08 & 0,04 \\
\hline 5 & 4,02 & 3,86 & 0,12 & 4,11 & 4,07 & 0,03 \\
\hline 6 & $4,0^{\prime}$ & 3,91 & 0,12 & 6,14 & 4,11 & 0,04 \\
\hline 7 & 4,05 & 3,93 & 0,10 & $4,1 ' t$ & 4,13 & 0,04 \\
\hline 24 & 4,32 & 4,19 & 0,05 & 4,35 & 4,31 & 0,01 \\
\hline 36 & 4,27 & 4,19 & 0,07 & 4,33 & 4,30 & 0,02 \\
\hline 48 & 4,32 & 4,18 & 0,07 & 4,39 & 4,33 & 0,01 \\
\hline 60 & 4,30 & 4,18 & 0,06 & 4,39 & 4,33 & 0,01 \\
\hline 72 & 4,14 & 4,13 & 0,09 & 4,37 & 4,20 & 0,05 \\
\hline 96 & 4,20 & 4,15 & 0,09 & 4,35 & 4,19 & 0,05 \\
\hline 108 & 4,13 & 4,06 & 0,09 & $t_{t}, 32$ & 4,17 & 0,05 \\
\hline
\end{tabular}

cpm : coups par minute.

cpm : counts per minute.

$\mathrm{dpm}:$ desintégrations/mn.

$\mathrm{dpm}$ : disintegrations/mn.

(1) voir, see, tableau, table 1.

\section{Excrétion de la radioactivité}

Les données expérimentales du tableau 3 ont montré que le maximum de radioactivité excrétée fut atteint 24 heures après l'administration des radioisotopes. Les résultats présentés dans le même tableau démontrent qu'il n'y a aucune différence statistique $(\mathrm{P}<0,05)$ pour la radioactivité excrétée, soit dans l'urine, soit dans les fèces des moutons défaunés ou non. En outre, il y a lieu de mentionner que la radioactivité excrétée par les fèces a dépassé celle des urines, résultats qui confirment ceux rapportés par EHLIG et coll. (I967). Rapportons également que de fortes variations individuelles ont été observées dans le taux total de radioactivité excrétée 


\section{TABLEAU 3}

Modalités de l'excrétion de la radioactivité chez le mouton ${ }^{+}$ après administration d'une seule dose de ${ }^{75}$ Se sélénométhionine ou ${ }^{3} \mathrm{H}$-sélénométhionine

Les valeurs sont données en logarithmes coups/mn ou $\mathrm{dpm}$ excrétion totale, urinaire ou fécale, avec l'erreur type

Total daily urinary and fecal excretion of radioactivity after administration to wethers ${ }^{+}$ by a stomach tube into the rumen or into the abomasum

of a single dose of ${ }^{75} \mathrm{Se}$ selenomethionine or ${ }^{3} \mathrm{H}$-selenomethionine

Values are mean logarithms of $\mathrm{cpm}$ or $\mathrm{dpm}$ of urine or feces with standard errors

\begin{tabular}{|c|c|c|c|c|c|c|}
\hline \multirow{2}{*}{$\begin{array}{cc}\text { Temps } & \text { Time } \\
\text { (heures) } & \text { (hours) } \\
\text { apres } & \text { after } \\
\text { administration }\end{array}$} & \multicolumn{3}{|c|}{${ }^{75}$ Se-sélénométhionine } & \multicolumn{3}{|c|}{${ }^{3} \mathrm{H}$-sélénométhionine } \\
\hline & $\begin{array}{l}\text { Sans infusoires } \\
\text { Defaunated cpm }\end{array}$ & $\begin{array}{l}\text { Holoxéniques } \\
\text { Control }\end{array}$ & $\begin{array}{l}\text { ET } \\
\text { SE }\end{array}$ & $\begin{array}{l}\text { Sans infusoires } \\
\text { Defaunated dpm }\end{array}$ & $\begin{array}{l}\text { Holoxéniques } \\
\text { Control }\end{array}$ & $\begin{array}{l}\text { ET } \\
\text { SE }\end{array}$ \\
\hline & \multicolumn{6}{|c|}{$\begin{array}{c}\text { Excrétion urinaire journalière } \\
\text { Daily Urinary Excretion }\end{array}$} \\
\hline 7 & 6,59 & 6,58 & 0,21 & 7,29 & 7,56 & 0,15 \\
\hline $2 ' t$ & 6,67 & 6,65 & 0,10 & 7,65 & 7,72 & 0,09 \\
\hline 36 & 6,36 & 6,15 & 0,04 & 7,13 & 7,17 & 0,10 \\
\hline 48 & $6,1,1$ & 6,26 & 0,09 & 7,30 & 7,33 & 0,07 \\
\hline 60 & 6,10 & $6,1_{1}$ & 0,16 & 7,10 & 6,90 & 0,10 \\
\hline $7:$ & 6,28 & 6,08 & 0,13 & 7,19 & 7,12 & 0,08 \\
\hline 96 & 6,05 & 5,82 & 0,07 & 6,76 & 7,19 & 0,25 \\
\hline 108 & 6,29 & 6,16 & 0,03 & 7,08 & 6,80 & 0,27 \\
\hline & \multicolumn{6}{|c|}{$\begin{array}{c}\text { Excrétion fécale journalière } \\
\text { Daily Fecal Excretion }\end{array}$} \\
\hline 7 & 5,40 & 5,50 & & & & \\
\hline 24 & $7,8: 2$ & 7,88 & & & & \\
\hline 36 & 7,50 & 7,60 & & & & \\
\hline 48 & 7,25 & 7,86 & & & & \\
\hline 60 & 7,17 & 7,27 & & & & \\
\hline 72 & 5,45 & 7,60 & & & & \\
\hline 96 & 6,96 & 6,98 & & & & \\
\hline 108 & 7,23 & 7,20 & & & & \\
\hline
\end{tabular}

Métabolisme du radioisotope dans le rumen

Durant les sept heures qui ont suivi l'administration orale du radioisotope environ $50 \mathrm{p}$. Ioo de la radioactivité enregistrée dans le jus du rumen a été observée dans la fraction protozoaire et les débris alimentaires (tabl. 4). Dans cette fraction et relativement aux autres fractions, la concentration de la radioactivité a progressivement augmenté pour atteindre 69 p. roo de celle du jus du rumen lors du dernier prélèvement de $96 \mathrm{~h}$. Il est à noter que le pic de la radioactivité dans le jus du rumen a été enregistré $z \mathrm{~h}$. après l'administration du radioisotope pour décliner par la suite continuellement. Dans la fraction soluble (ATA bactérienne) on a constaté, lors du prélèvement de $5 \mathrm{~h}$. une importante concentration atteignant jusqu'à $2 \mathrm{I}$ p. IoO 
TABLEAU 4

Métabolisme de la ${ }^{75}$ Se-L-sélénométhionine dans le rumen du mouton (')

Metabolism of the ${ }^{75}$ Se-L-selenomethionine in the sheep's rumen

\begin{tabular}{|c|c|c|c|c|}
\hline \multirow{3}{*}{\begin{tabular}{c} 
Temps \\
(heures) (hours) \\
après after \\
administration \\
\multicolumn{2}{c}{${ }^{75 e}$}
\end{tabular}} & \multirow{3}{*}{$\begin{array}{c}\text { Coups } / \mathrm{mn} / \mathrm{ml} \\
\text { Jus rumen }(\mathrm{JR}) \\
\text { counts/mn/ml } \\
\text { rumen liquor (RL) }\end{array}$} & \multicolumn{3}{|c|}{$\begin{array}{c}\text { Radioactivité (\%) dans les fractions JR } \\
\text { Radioactivity (\%) in RL fractions }\end{array}$} \\
\hline & & \multicolumn{2}{|c|}{ Bactéries } & \multirow{2}{*}{$\begin{array}{c}\text { Protozoa } \pm \text { débris } \\
\text { alimentaires } \\
\text { plant particles }\end{array}$} \\
\hline & & $\begin{array}{l}\text { ATA insoluble } \\
\text { TCA precipitated }\end{array}$ & $\begin{array}{l}\left.\text { ATA soluble ( }{ }^{2}\right) \\
\text { TCA soluble }\end{array}$ & \\
\hline 1 & $9597 \pm 7262$ & 31 & 16 & 53 \\
\hline 2 & $18020 \pm 1562$ & 31 & 16 & 55 \\
\hline 3 & $14715 \pm 5055$ & 25 & 20 & 55 \\
\hline 4 & $11337 \pm 2958$ & 29 & 20 & 51 \\
\hline 5 & $10918 \pm 2261$ & 30 & 21 & 48 \\
\hline 6 & $11009 \pm 2451$ & 29 & 19 & 51 \\
\hline 7 & $11865 \pm 3645$ & 26 & 19 & 55 \\
\hline 24 & $5777 \pm 1533$ & 25 & 15 & 55 \\
\hline 36 & $3147 \pm \quad 779$ & 25 & 14 & 61 \\
\hline 48 & $2266 \pm \quad 783$ & 23 & 12 & 64 \\
\hline 60 & $1451 \pm \quad 570$ & 22 & 12 & 66 \\
\hline 72 & $1382 \pm 948$ & 21 & 12 & 68 \\
\hline 84 & $421 \pm$ & 21 & 12 & 67 \\
\hline 96 & $369 \pm$ & 20 & 11 & 69 \\
\hline
\end{tabular}

(1) Cinq moutons de 30-35 kg ont reçu par 2 voies orales une seule dose de ${ }^{75} \mathrm{Se}(7,14 \mu \mathrm{Ci} / \mathrm{kg}$ poids vif). Five sheep $30-35 \mathrm{~kg}$ were administered with one single oral dose of ${ }^{75} \mathrm{Se}$.

\pm Erreur standard de la moyenne. \pm standard deviation of the mean.

ATA : acide trichloroacétique. TCA : trichloroacettic acid.

(2) Ceci inclut également la fraction non cellulaire (2-3 p. 100), obtenue après centrifugation du jus du Rumen à $30000 \times g$.

de l'activité totale du jus du rumen. Une importante concentration de radioactivité allant jusqu'à $30 \mathrm{p}$. Ioo de l'activité totale du jus du rumen a été observée dans l'insoluble ATA bactérien. Ce pourcentage est inférieur aux résultats obtenus par Paulson, Baumann et Pope (I968) qui ont mis en évidence une incorporation de l'ordre de $68 \mathrm{p}$. Ioo dans l'ATA insoluble des microorganismes du rumen lors de leur incubation in vitro en présence de ${ }^{75}$ Ses-élénométhionine. Après l'hydrolyse acide de l'insoluble ATA bactérien, 50 p. roo de l'activité a été mesurée dans un résidu noirâtre qui resta adhéré au tube d'hydrolyse. C'est également la constatation de Sternberg et Mercier (I964) dans le cas de l'hydrolyse des bactéries BCG incubées avec du ${ }^{75} \mathrm{SeO}_{3} \mathrm{H}_{3}$. D'après ces deux auteurs, il est possible qu'il s'agisse là d'une précipitation de sélénium suivie d'une adsorption de surface sur les résidus noirs après hydrolyse. Suite à l'hydrolyse enzymatique à la pronase, cinq bandes radioactives ont été décelées sur les chromatogrammes monodimensionnels des protéolysats bactériens séparés par l'emploi du système-solvant (1) ci-après : $n$-buta- 
nol-pyridine-eau (I : I : I v/v/v); I8 p. Ioo de la radioactivité a été mesurée au lieu d'origine, $50 \mathrm{p}$. Ioo à été retrouvée dans une bande avec un $\mathrm{Rf} 0,87$. Cette bande n'a pas pu être identifiée, $20 \mathrm{p}$. Ioo de la radioactivité a été récupérée dans une bande dont le $\mathrm{Rf}(0,65)$ était similaire à celle de la sélénométhionine. Le reste de la radioactivité a été distribué entre deux bandes non identifiées, situées audessus et au-dessous de la bande de ${ }^{75}$ Se-sélénométhionine. Cette dernière a été rechromatographiée avec de la sélénométhionine authentique en se servant des deux systèmes de solvants, solvant $I$ et solvant $2: n$-butanol-acide acétique-eau (25:6:25 $\mathrm{v} / \mathrm{v} / \mathrm{v})$. Par cette cochromatographie bidimensionnelle, I 8 p. Ioo de la radioactivité originale des protcolyats chromatographiés a été retrouvée comme ${ }^{75}$ Se-sélénométhionine. Des autoradiographies ont d'ailleurs confirmé ceci. Suite aux chromatographies sur papier des protolysats des bactéries incubées du rumen avec de 1a ${ }^{75} \mathrm{Se}-$ sélénométhionine obtenues par hydrolyse enzymatique, PAUL, BAUMANN et POPE (I968) ont récupéré de 57 à 62 p. Ioo de la radioactivité des hydrolysats dans la sélénométhionine. Les écarts des taux de la radioactivité incorporée dans la sélénométhionine que nous avons observé et celle de PaUison, BaUManN et PoPe (I968) peuvent être dus à une différence dans le métabolisme entre in vivo et in vitro de cet acide aminé.

\section{CONCL USION}

Du fait que la sélénométhionine prédomine sur toutes les autres formes de sélénium existant dans les fourrages, (PETERSON et BUTLER, I969) cela nous a incité à étudier son métabolisme dans le rumen, sa distribution tissulaire et son excrétion, et toutes ces différentes analyses ont été exécutées au moyen de sélénométhionine radioactive. Des études destinées à déterminer l'absorption de la sélénométhionine au niveau de l'intestin du ruminant sont présentement en cours.

Reçu pour publication en mai 1973.

\section{SUMMARY}

EFFECT OF DEPARASITISM ON THE USE OF SELENOMETHIONINE IN SHEEP

\footnotetext{
Study of the use of selenomethionine in sheep, deparasitized or not, is done after ${ }^{75}$ Se or ${ }^{3} \mathrm{H}$ labelling. Twenty sheep are divided into 2 groups of to each - the first is composed of normal individuals, and the second of deparasitzed animals. Deparasitism is carried out by oral administration of sodium dioctylsulphosuccinate for 6 consecutive days, which completely eliminates protozoa after the first 3 days of treatment. In each group of ro animals, 6 received ${ }^{75} \mathrm{Se}$ and 4 tritium. There is no difference in distribution of tissue radioactivity and its excretion due to deparasitism. Maximal radioactivity is recorded in kidneys, liver, pancreas; high specific activity is also recorded in some parts of the digestive tract. After administration of radioselenium, plasma radioactivity peak occurs at 24 hours for $\mathrm{Se}^{75}$ and 60 hours for tritium ; maximal urinary radioactivity appears 24 hours after ingestion of radioisotopes. More radioactivity is excreted by feces than by urine.
} 


\section{RÉFÉRENCES BIBIIOGRAPHIQUES}

Abou Akmada A. R., Barteey E. E., Berube R., Fina L. R., Meyer R. M., Hendricks D., Julius F., Ig68. Simple method to remove completely ciliate protozoa of adult ruminant. Appl. Microbiol., 16, I475-I477.

Abou Akkada A. R., El-Shazly, 1965. Effect of presence or absence of rumen ciliate protozoa on some blood components, nitrogen retention and digestibility of food constituents in lambs. J. Agric. Sci., 64, $25 \mathrm{I}-255$.

Coleman G. S., 1967. The metabolism of free amino acids by washed suspensions of rumen ciliate Entodinium candatum. J. Gen. Microbiol., 47, 433-447.

Conti L., Hegesippe S. De Saint-Sauveur, Tempe J., 1972. Biosynthèse de sélénométhionine-75e pour usage scintigraphique. Int. J. Appl. Rad. Isot., 23, 4I I-421.

Dawson R. M. C., Kemp P., I969. The effect of defaunation on the phospholipids and on the hydrogenation of unsaturated fatty acids in the rumen. Biochem. J., 115, 35 I-352.

Ehlig C. F., Hogue D. E., Allaway W. H., Hamm D. J., ig67. Fate of selenium from selenite or seleno-methionine with or without vitamin E, in lambs. J. Nutr., 92, I 2 I-126.

HansSON E., JacoBSon S. O., 1966. Uptake of $/{ }^{75} \mathrm{Se} /$ selenomethionine in the tissues of the mouse studied by whole-body autoradiography. Biochim. Biophys. Acta, 115, 285-293.

Harmeyer J., Hill H., I965. The biosynthesis of amino acids by isolated rumen protozoa species. p. 95-I09. In : Radioisotopes in animal nutrition and physiology. International Atomic Agency, Vienna.

HedegaArd J., Falcone G., Calabro S., I963. Incorporation de selenium dans des analogues des acides aminés soufrés, dans Candida albicans. C. R. Soc. Biol., 157, 280-284.

Hidiroglou M., Heaney D. P., Jenkins J. K., 1968. Metabolism of inorganic selenium in rumen bacteria. Can. J. Physiol. Phamacol., 46, 229-232.

Hungate R. E., I950. Mutualism in protozoa. Ann. Res. Microbiol., 4, 53-66.

JacoBson S. O., 1966. Uptake of ${ }^{75} \mathrm{Se}$ in tissues of sheep after administration of a single dose of ${ }^{75} \mathrm{Se}$ sodium ${ }^{75}$ Se-sodium selenite, ${ }^{75}$ Se-selenomethionine of ${ }^{75}$ Se-selenocystine. Acta. Vet Scand., 7, I-I5.

JENKINS K., Hidiroglou M., 1967. The incorporation of ${ }^{75}$ Se-selenite into dystrophogenic pasture grass. The chemical nature of the selenocompounds formed and their availability to young ovine. Can. J. Biochem., 45, 1027-1040.

Lindsay J. R., Hogan J. P., I972. Digestion of two legumes and rumen bacterial growth in defaunated sheep. A ust. J. A gric. Res., 23, 32I-330.

Neethling L. P., Brown J. M. M., Osterhoff D. R., de Wett P. J., Wardcox I. S., I968. A note on an abnormal haemoglobin in cases of the gceldikkop-enzootic icterus disease cowplen tl. S.Afr. vet. Ass., 39, 25-33.

Patterson M. S., Green R. C., I965. Measurement of low energy beta-emitters in aqueous solution by liquid scintillation counting of emulsions. Analyt. Chem., 37, 854-857.

Paulson G. D., Baumann, Pope A. L., I968. Metabolism of ${ }^{75}$ Se-selenite, ${ }^{75}$ Se-selenate, ${ }^{75}$ Se-selenomethionine and ${ }^{35}$ S-sulfate by rumen microorganisms in vitro. J. Anim. Sc., 27, 497-504.

Peterson P. J., Butler., I962. The uptake and assimilation of selenite by higher plants. Aust. J. Biol. Sc., 15, I $26-146$.

Sauvant D., Gouet P., I970. Comparaisons de deux techniques pour dénombrer les protozoaires du rumen et précision obtenue. Ann. Biol. anim. Bioch. Biophys., 10, 689-696.

Sternberg J., Mercier A., I964. Étude comparative sur le marquage de microorganisms par le selenium-75. Int. J. Appl. Rad. and. Isotop, 15, 587-598.

Snedecor G. W., Cochran W. G., I967. p. 329 Statistical methods. The Iowa State University Press, Ames, Iowa.

Weller R. A., Gray F. V., Pilgrim A. F., I958. The conversion of plant nitrogen to microbial nitrogen in the rumen of the sheep. Brit. J. Nutr., 12, 42I-429.

Williams P. P., Davies R. E., Doetsch R. N., Gutierrez J., Ig6r. Physiological studies of the rumen protoan Ophryoscolex caudatus EBerLeIn. Appl. Microbiol., 9, 405-409.

WRight, D. E., Hungate R. E., 1967. Metabolism of glycin by rumen microorganisins. Appl. Microbiol., 15, 152-157. 